\title{
Adult and paediatric size bronchoscopes for bronchoalveolar lavage in mechanically ventilated patients: yield and side effects
}

\author{
Bara Ricou, Stéphane Grandin, Laurent Nicod, Jean-Benoît Thorens, Peter M Suter
}

\begin{abstract}
Background - Bronchoalveolar lavage is considered a safe procedure. When performed in the mechanically ventilated patient, however, potentially harmful effects on respiratory and haemodynamic functions have been reported in which the size of the bronchoscope may play a part. Two different size bronchoscopes (adult and paediatric) were therefore investigated with regard to bronchoalveolar lavage yield and side effects.

Methods - Twenty mechanically ventilated patients underwent bronchoalveolar lavage with both adult and paediatric bronchoscopes in a randomised sequential manner.

Results - In a total of 45 pairs of bronchoalveolar lavage procedures no difference was noted between adult and paediatric bronchoscopes with regard to total cell yield, differential cell count, and microbiological results. Peak intratracheal pressure increased with the adult bronchoscope only. Systemic arterial pressures increased more with the adult than with the paediatric bronchoscope. $\mathrm{PaO}_{2}$ decreased with the adult but not with the paediatric bronchoscope.

Conclusions - The paediatric bronchoscope offers a comparable bronchoalveolar lavage yield in mechanically ventilated patients to the adult bronchoscope, while the respiratory and haemodynamic side effects are significantly lower than with the adult size instrument.
\end{abstract}

Intensive Care

B Ricou

$S$ Grandin

P M Suter

Division of

Pneumology

L Nicod

J B Thorens

Departments of Anesthesiology and Medicine,

University Hospital of Geneva,

1211 Genève 14, Switzerland

Reprint requests to: Dr B Ricou.

Received 13 June 1994 Returned to authors 5 September 1994 Revised version received 6 October 1994

Accepted for publication 16 November 1994

Bronchoalveolar lavage has increased the use of the bronchoscope in critical care medicine by sampling cells and non-cellular components which are representative of inflammatory events in the lower respiratory tract. ${ }^{1-3}$ Moreover, it is a useful technique in nosocomial pneumonia, a frequent complication during mechanical ventilation, by providing samples for microbiological testing. ${ }^{4}$

Although bronchoalveolar lavage seems safe even in patients with adult respiratory distress syndrome, ${ }^{5}$ respiratory and cardiovascular complications have been reported in the critically ill. ${ }^{67}$ These complications seem to depend
(Thorax 1995;50:290-293)

on the relation between the diameter of the endotracheal tube and the size of the bronchoscope. ${ }^{6}$ To overcome these risks it has been proposed that smaller bronchoscopes or small lavage fluid volumes be employed. ${ }^{58}$ However, no studies have to date confirmed that these techniques are safer. More importantly, it is not known whether a small volume of instilled lavage fluid gives the same yields as more traditional volumes of bronchoalveolar lavage fluid with regard to the recovery of fluid and cell components.

The purpose of this study was to compare the yield of bronchoalveolar lavage fluid and the effects on ventilatory and haemodynamic function and pulmonary gas exchange of two bronchoscopes of different sizes, one adult with an outer diameter of $5.9 \mathrm{~mm}$ and a paediatric model with an outer diameter of $3.4 \mathrm{~mm}$.

\section{Methods}

Twenty mechanically ventilated patients admitted to our surgical intensive care division after trauma or major surgery and requiring bronchoscopy for diagnosis of pneumonia were studied. Exclusion criteria were $\mathrm{PaO}_{2}$ below $75 \mathrm{~mm} \mathrm{Hg}(10 \mathrm{kPa})$ at an $\mathrm{FIO}_{2}$ of $1 \cdot 0$, peak intratracheal pressure above $50 \mathrm{~cm} \mathrm{H}_{2} \mathrm{O}$, severe haemodynamic instability, and an endotracheal tube diameter of $\leqslant 7 \mathrm{~mm}$.

Each patient underwent two bronchoscopies in a randomised sequential manner using a standard adult size bronchoscope (Olympus BF type 30; outer diameter $5.9 \mathrm{~mm}$ ) and a paediatric size bronchoscope (Pentax FB 10X; outer diameter $3.4 \mathrm{~mm}$ ). The two lavages were performed in the same segment - that is, lingula or right middle lobe - in each patient according to standard techniques. ${ }^{1}$ With the adult bronchoscope saline was instilled at a volume of $2 \times 50 \mathrm{ml}$ and with the paediatric bronchoscope the volume used was $2 \times 25 \mathrm{ml}$, both at room temperature.

Ventilator settings and drug infusions were kept unchanged throughout the procedure. Minute ventilation, tidal volume, peak intratracheal pressure, positive end expiratory pressure, and respiratory rate were measured at baseline, during bronchoscopy and after the procedure. Haemodynamic variables and pulse oximetry were monitored continuously throughout. Arterial blood gases were analysed before, at the end of, and 15 minutes after bronchoscopy. Native bronchoalveolar lavage results were tested for total cell numbers, differential cell count and pathogens. Protein 

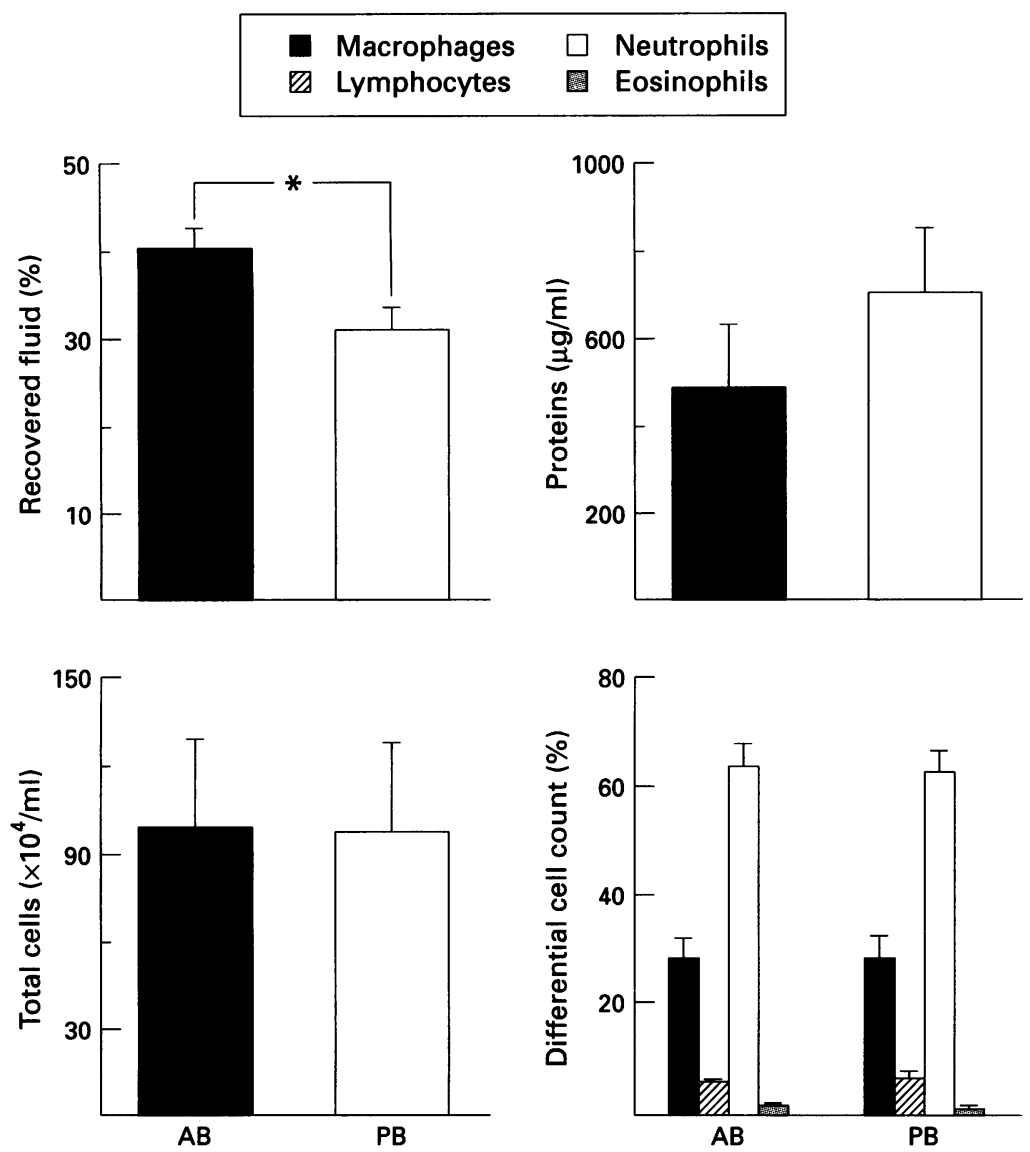

Figure 1 Bronchoalveolar lavage yields from mechanically ventilated patients using adult $(A B)$ and paediatric (PB) bronchoscopes. Mean values $\pm 1 S E$ are indicated. ${ }^{*} p<0.05$ adult versus paediatric bronchoscope.

concentrations were measured in cell free supernatants.

\section{STATISTICAL ANALYSIS}

The results are given as mean (SE) values. Statistical analysis was performed using the Student's two tailed unpaired $t$ test for bronchoalveolar lavage cell and fluid returns, two way analysis of variance for repeated measures when considering variables before, during and after bronchoscopy, and factorial analysis when comparison was made between the adult and paediatric bronchoscope groups.

\section{Results}

A total of 45 pairs of bronchoalveolar lavage procedures was performed in 20 patients. The sequence did not influence the findings and the results are therefore expressed globally for adult and paediatric bronchoscopy groups. The mean age of the patients was 51 years (range 19 to 86$)$.

YIELD OF BRONCHOALVEOLAR LAVAGE FLUID Total cell numbers, differential cell count, percentage of recovered fluid, and protein concentration are summarised in fig 1 . The microbiological findings were similar for both techniques. Gram stain and microbiological culture results were identical in $86 \%$ and $94 \%$ of pairs respectively.
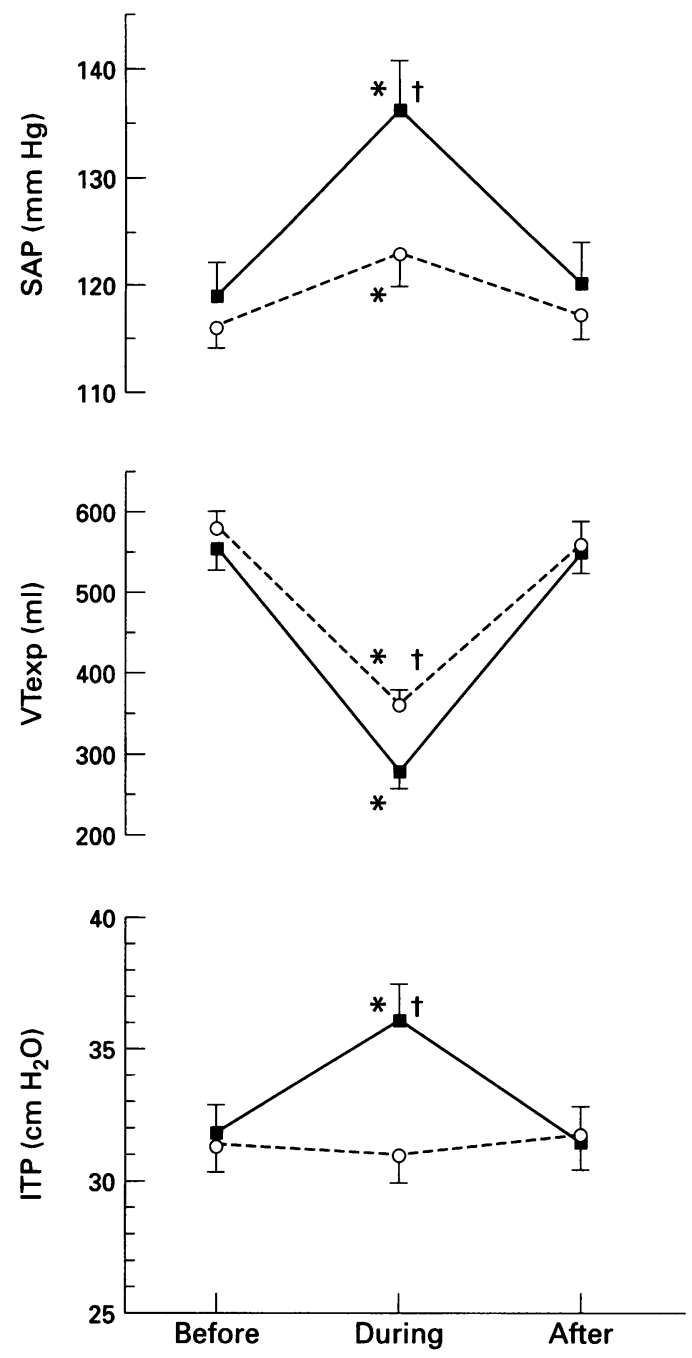

Figure 2 Mean (SE) cardiovascular and respiratory variables before, during and after adult ( $A B,-\square-)$ or paediatric $(P B,-\bigcirc-)$ bronchoscopy. $S A P=$ systolic arterial pressure; VTexp = expiratory tidal volume; ITP $=$ peak intratracheal pressure. ${ }^{*} p<0.05$ before versus during the procedure; $\dagger p<0.05$ adult versus paediatric bronchoscope.

RESPIRATORY AND HAEMODYNAMIC CHANGES

Figure 2 summarises changes in respiratory and haemodynamic indices during and after bronchoalveolar lavage. The difference in intratracheal pressure between adult and paediatric bronchoscopy was significant only when an endotracheal tube of $<9 \mathrm{~mm}$ internal diameter was used $\left(42 \cdot 7(2 \cdot 2)\right.$ v $33.7(1 \cdot 6) \mathrm{cm} \mathrm{H}_{2} \mathrm{O}$, $\mathrm{p}<0.05)$. Expiratory tidal volume was lower with the adult bronchoscope $(p<0 \cdot 05)$. During the procedure minute ventilation decreased with the adult and paediatric bronchoscope by $46 \%$ and $38 \%$ respectively $(p<0 \cdot 05)$. Respiratory rate increased significantly with the adult bronchoscope from $22(1 \cdot 2)$ to $25(1 \cdot 6)$ breaths/min $(\mathrm{p}<0.05)$, but did not change with the paediatric bronchoscope. The heart rate did not change significantly. No arrhythmias were noted. Alterations in gas exchange during the bronchoalveolar lavage are illustrated in fig 3. Significant changes between pre and post bronchoscopy measurements were observed using the adult but not the paediatric bronchoscope. No significant hypoxaemia persisted for more than 30 minutes after the procedure. 

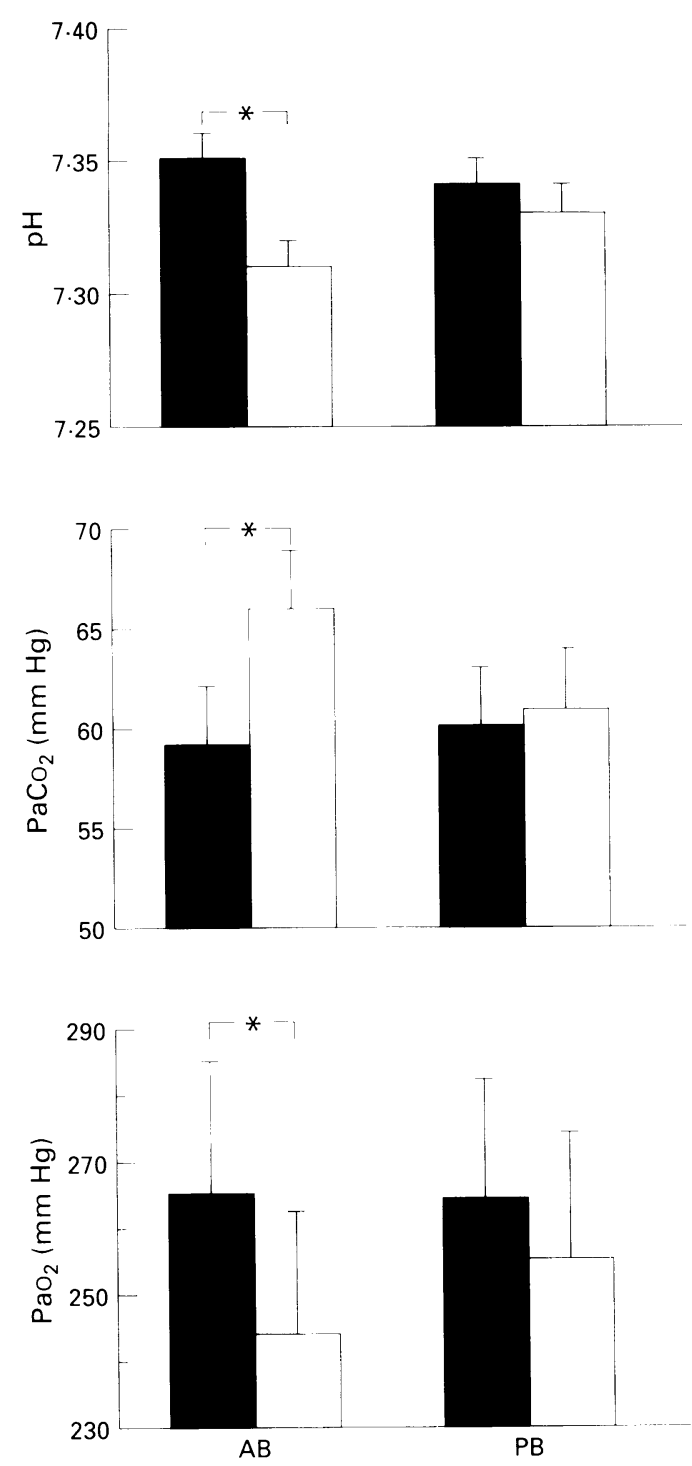

Figure 3 Gas exchange in mechanically ventilated patients undergoing bronchoscopy using an adult (AB) or paediatric bronchoscope (PB). ${ }^{*} p<0.05$ before ( $\square$ versus after ( $\square$ ) the procedure.

Neither pneumothorax nor haemorrhage complicated either procedure.

\section{Discussion}

Our study shows similar yields of recovered fluid and cells for the two bronchoscopes in patients requiring mechanical ventilation for acute lung failure. Differential and total cell counts were comparable, suggesting that lavage with a paediatric bronchoscope samples the same lining fluid of the air spaces as conventional lavage with an adult bronchoscope. The percentage of recovery of the instilled fluid was lower with the paediatric bronchoscope than with the adult instrument, but this is consistent with the observations of Baldwin et al who obtained $17 \%$ returns following lavage with $20 \mathrm{ml}^{8}$

Our data confirm that secondary effects of fibreoptic bronchoscopy and bronchoalveolar lavage on respiratory and cardiovascular function depend essentially on the degree of endotracheal tube obstruction by the broncho- scope. With an endotracheal tube of $8 \mathrm{~mm}$ diameter the adult bronchoscope occupies about $54 \%$ of the cross-sectional area and the paediatric bronchoscope only $18 \%$. The intratracheal pressure increased more with the adult bronchoscope than with the paediatric instrument with an endotracheal tube of $8 \mathrm{~mm}$. However, it does not seem to be influenced by an obstruction of less than $50 \%$ since the pressure did not increase with either the adult or paediatric bronchoscope when an endotracheal tube of $9 \mathrm{~mm}$ internal diameter was used.

The decrease in tidal and minute volumes during bronchoscopy can be due to two factors: (1) higher intratracheal pressure causing a leak in the ventilatory tubing system, and (2) suctioning. The adult bronchoscope caused a higher intratracheal pressure and a greater decrease in tidal volume, suggesting a greater leakage. The resulting decrease in alveolar ventilation induced a rise in $\mathrm{PaCO}_{2}$ and a decrease in $\mathrm{pH}$ with the adult bronchoscope. Most of these patients were ventilated with permissive hypercapnia which explained the increased baseline $\mathrm{PaCO}_{2}$. The increase in $\mathrm{PaCO}_{2}$ during bronchoscopy resulted in no observable harmful effect in our study group. Some studies have specifically addressed the effects of bronchoscopy on gas exchange in mechanically ventilated patients ${ }^{57}$ and have reported a variation in $\mathrm{PaO}_{2}$ from $+10 \%$ to $-30 \%$ depending on the severity of pulmonary dysfunction. Although in our study the overall decrease in $\mathrm{PaO}_{2}$ was moderate with the adult bronchoscope $(-8 \%)$, it was significantly higher than with the paediatric instrument. This fall in $\mathrm{PaO}_{2}$ may be due to the decreased tidal volume as well as to the fluid instilled in the alveolar compartment."

The cardiovascular side effects with the adult bronchoscope were not significantly different from those with the paediatric instrument, with no clinically significant arrhythmias or excessive increases in blood pressure such as those reported elsewhere. ${ }^{79}$ The increases in systolic arterial pressure were, however, significantly higher with the adult bronchoscope. Finally, fibreoptic bronchoscopy appeared to be better tolerated with the paediatric bronchoscope; patients were less agitated, coughed less, and looked more comfortable than with the adult instrument, important considerations in the intensive care unit where patients are subjected to aggressive diagnostic and therapeutic procedures.

The results of this study suggest that a paediatric bronchoscope with a $3 \cdot 4 \mathrm{~mm}$ outer diameter presents definite advantages over an adult sized bronchoscope in the mechanically ventilated patient, with less significant cardiovascular and respiratory side effects while maintaining comparable cell and microbiological yields. The use of the smaller bronchoscope should be considered particularly in higher risk patients such as those with coronary artery disease or severe pulmonary gas exchange impairment.

This work was supported by grant nos 32.33902 .92 and 31.31019 .91 from the Swiss National Research Foundation 
(FNRS) and grant no 1830 from the Fondation pour Recherches Médicales Carlos et Elsie De Reuter.

1 Reynolds H. Bronchoalveolar lavage. Am Rev Respir Dis 1987; 135:250-63.

2 Jolliet Ph, Chevrolet JC. Bronchoscopy in the intensive care unit. Intensive Care Med 1992;18:160-9.

3 Suter P, Suter S, Girardin E, Roux-Lombard P, Grau GE, Dayer JM. High bronchoalveolar levels of tumor necrosis factor and its inhibitors, interleukin-1, interferon and elastase in patients with adult respiratory distress syndrome after trauma, shock or sepsis. Am Rev Respir Dis 1992;145: 1016-22.

4 Torres A, Puig de la Bellacasa J, Xobet A. Diagnostic value of quantitative cultures of bronchoalveolar lavage and telescoping plugged catheters in mechanically ventilated patients with bacterial pneumonia. Am Rev Respir Dis 1989; 140:306-10.

5 Steinberg KP, Mitchell DR, Maunder RJ, Milberg JA, Whitcomb ME, Hudson LD. Safety of bronchoalveolar lavage in patients with adult respiratory distress syndrome. $A m$ Rev Respir Dis 1993;148:556-61.

6 Lindholm C, Ollman B. Cardiorespiratory effects of flexible fiberoptic bronchoscopy in critically ill patients. Chest 1978; 74:362-7.

7 Trouillet JL, Guiget $M$. Fiberoptic bronchoscopy in ventilated patients. Evaluation of cardiopulmonary risk under mipatients. Evaluation of cardiopulmonary

8 Baldwin DR, Wise R, Andrews JM, Honeybourne D. Microlavage: a technique for determining the volume of epithelial lining fluid. Thorax 1991;46:658-62.

9 Burns D, Shure D, Moser K. The physiologic consequences of saline lobar lavage in healthy human adults. Am Rev Respir Dis 1983;127:695-701.

\section{Adventitia}

\section{The "Cochrane Collaboration"}

The current publicity given to the "Cochrane Collaboration" calls attention to the many contributions made by the late Archie Cochrane to the medical and scientific communities. He and Bradford Hill were the early proponents of the randomised clinical trial. Both cogently argued for its adoption at a time when the inclusion of controls or referents in clinical trials was almost unknown in North America. The idea of a series of centres reviewing various clinical trials and finding whether their design was acceptable and the results valid would probably have been received by Archie with lukewarm enthusiasm. Those who knew him feel that, although he would endorse the need to analyse the data included in such trials, with few exceptions he would have found the task burdensome and mundane.

Unlike most of the participants in the Cochrane Collaboration, Archie was a "shoe leather" epidemiologist who went out and about. He and Ian Higgins stomped all over the Rhondda Fach, visiting house after house, pleading for participation so that they could administer his questionnaire, perform or supervise spirometric tests, and take over 2000 ECG tracings in homes or village halls. They performed similar feats in Stavely, Derbyshire where they interviewed steel workers, coal miners and those who worked for chemical companies, mostly in Derbyshire drizzle and heavy air pollution. Those passing through Stavely in the 1950 s, while realising that it was not the end of the world, certainly thought they could see off the edge!

Archie did not take himself too seriously and was singularly unimpressed by the medical establishment. While he held the Chair of Tuberculosis in Cardiff he tried to persuade the local cardiologists to carry out a clinical trial involving the treatment of myocardial infarction at home and in hospital. His suggestion was rejected somewhat vehemently, but somehow Archie managed to persuade a cardiologist in Bristol to go along with the idea and subsequently he presented his findings to the British Heart Association. He told the audience that those who were treated in hospital had a slightly, but not significantly, better survival rate than those who were treated at home. This prompted a member of the audience to tell him that the trial was unethical and may have led to unnecessary deaths since it was obvious that all patients with a myocardial infarction should be admitted to hospital. Archie then announced, with typical Cochrane panache, that he had deliberately interchanged the results and that those who had been treated at home had done slightly, but not significantly, better. His prior interrogator was then asked whether this meant that the admission to hospital of any patient with a myocardial infarction constituted negligence.

At about the same time Ian Higgins presented his data on the interpretation of over 2000 ECGs performed on the inhabitants of the Rhondda Fach. He pointed out that, with one interpretation around $25 \%$ of the ECGs were abnormal, but if two interpreters were used and there had to be agreement, the number of abnormal ECGs fell to $16 \%$. Increasing the number of interpreters led to fewer and fewer ECGs being agreed as abnormal until with seven or eight readers all the ECGs would have been interpreted as normal. This led to the formulation of Higgins' Law: "The apparent frequency of any condition is inversely proportional to the number of investigators (or investigations) required to establish its frequency."

I suspect Archie would feel that the Cochrane Collaborators who stay in their office and analyse the various studies are somewhat akin to Sassoon's "Scarlet Majors at the Base who speed glum heroes up the line, if not to death" but to endless tedium. 